TRANSACTIONS OF THE

AMERICAN MATHEMATICAL SOCIETY

Volume 361, Number 9, September 2009, Pages 4561-4580

S 0002-9947(09)04763-1

Article electronically published on April 13, 2009

\title{
ASYMPTOTIC STABILITY OF THE WAVE EQUATION ON COMPACT SURFACES AND LOCALLY DISTRIBUTED DAMPING-A SHARP RESULT
}

\author{
M. M. CAVALCANTI, V. N. DOMINGOS CAVALCANTI, R. FUKUOKA, \\ AND J. A. SORIANO
}

\begin{abstract}
This paper is concerned with the study of the wave equation on compact surfaces and locally distributed damping, described by

$$
\left.u_{t t}-\Delta_{\mathcal{M}} u+a(x) g\left(u_{t}\right)=0 \quad \text { on } \mathcal{M} \times\right] 0, \infty[,
$$

where $\mathcal{M} \subset \mathbb{R}^{3}$ is a smooth oriented embedded compact surface without boundary. Denoting by $\mathrm{g}$ the Riemannian metric induced on $\mathcal{M}$ by $\mathbb{R}^{3}$, we prove that for each $\epsilon>0$, there exist an open subset $V \subset \mathcal{M}$ and a smooth function $f: \mathcal{M} \rightarrow \mathbb{R}$ such that meas $(V) \geq \operatorname{meas}(\mathcal{M})-\epsilon$, Hessf $\approx \mathbf{g}$ on $V$ and $\inf _{x \in V}|\nabla f(x)|>0$.

In addition, we prove that if $a(x) \geq a_{0}>0$ on an open subset $\mathcal{M} * \subset \mathcal{M}$ which contains $\mathcal{M} \backslash V$ and if $g$ is a monotonic increasing function such that $k|s| \leq|g(s)| \leq K|s|$ for all $|s| \geq 1$, then uniform and optimal decay rates of the energy hold.
\end{abstract}

\section{INTRODUCTION}

Let $\mathcal{M}$ be a smooth oriented embedded compact surface without boundary in $\mathbb{R}^{3}$ and let $\mathbf{g}$ denote the Riemannian metric induced on $\mathcal{M}$ by $\mathbb{R}^{3}$. For $\epsilon>0$ we prove that there exist an open subset $V \subset \mathcal{M}$ and a smooth function $f: \mathcal{M} \rightarrow \mathbb{R}$ such that meas $(V) \geq \operatorname{meas}(\mathcal{M})-\epsilon$, Hessf $\approx \mathbf{g}$ on $V$ and $\inf _{x \in V}|\nabla f(x)|>0$ (see Subsection 4.4).

We denote by $\nabla_{T}$ the tangential gradient on $\mathcal{M}$ and by $\Delta_{\mathcal{M}}$ the Laplace-Beltrami operator on $\mathcal{M}$. This paper is devoted to the study of the uniform stabilization of solutions of the following damped problem:

$$
\begin{cases}u_{t t}-\Delta_{\mathcal{M}} u+a(x) g\left(u_{t}\right)=0 & \text { on } \mathcal{M} \times] 0, \infty[, \\ u(x, 0)=u^{0}(x), \quad u_{t}(x, 0)=u^{1}(x) & x \in \mathcal{M},\end{cases}
$$

where $a(x) \geq a_{0}>0$ on an open proper subset $\mathcal{M}_{*} \supset \mathcal{M} \backslash V$ of $\mathcal{M}$ and in addition $g$ is a monotonic increasing function such that $k|s| \leq|g(s)| \leq K|s|$ for all $|s| \geq 1$.

A natural question arises in the context of the wave equation on compact surfaces: Would it be possible to stabilize the system by considering a localized feedback acting only on a portion of the surface? In the affirmative case, what would be the

Received by the editors April 26, 2007.

2000 Mathematics Subject Classification. Primary 32J15, 35L05, 47J35, 93 D15.

Key words and phrases. Compact surfaces, wave equation, locally distributed damping.

The research of the first author was partially supported by the CNPq Grant 300631/2003-0.

The research of the second author was partially supported by the CNPq Grant 304895/2003-2.

(C)2009 American Mathematical Society Reverts to public domain 28 years from publication 
geometrical impositions we have to assume on the surface? When the damping term acts on the whole surface, the conjecture was studied by Cavalcanti and Domingos Cavalcanti in CA-DO and also by Andrade et al. in An1, An2 in the context of viscoelastic problems. For linear waves, we can mention the works due to Rauch and Taylor Ra-Ta, Hitrik [HIT and, recently Christianson [CHR. For the nonlinear wave equation on compact manifolds with boundary, it is important to cite the work due to Triggiani and Yao TRI-YAO]. More recently, the authors of the present work Ca-Do-Fu-So studied the linear wave equation on a compact surface $\mathcal{M}$ without boundary supplemented with a nonlinear and localized dissipation. In this article the authors prove the above conjecture when the portion of $\mathcal{M}$ where the damping is effective is strategically chosen. Setting $\mathcal{M}=\mathcal{M}_{0} \cup \mathcal{M}_{1}$, where

$$
\mathcal{M}_{1}:=\{x \in \mathcal{M} ; m(x) \cdot \nu(x)>0\} \text { and } \mathcal{M}_{0}=\mathcal{M} \backslash \mathcal{M}_{1},
$$

$m(x):=x-x^{0}\left(x^{0} \in \mathbb{R}^{3}\right.$ fixed $)$ and $\nu$ is the exterior unit normal vector field of $\mathcal{M}$, then for $i=1, \ldots, k$, they assume that there exist open subsets $\mathcal{M}_{0 i} \subset \mathcal{M}_{0}$ of $\mathcal{M}$ with smooth boundary $\partial \mathcal{M}_{0 i}$ such that the $\mathcal{M}_{0 i}$ are umbilical. Moreover, they suppose that the mean curvature $H$ of each $\mathcal{M}_{0 i}$ is nonpositive (i.e. $H \leq 0$ on $\mathcal{M}_{0 i}$ for every $\left.i=1, \ldots, k\right)$ and that the damping is effective on an open subset $\mathcal{M}_{*} \subset \mathcal{M}$ which contains $\mathcal{M} \backslash \bigcup_{i=1}^{k} \mathcal{M}_{0 i}$. Roughly speaking, the region which does not contain dissipative effects must be umbilical. This is required since the authors employ the same multipliers considered in solving the similar question for the wave equation,

$$
u_{t t}-\Delta u+a(x) g\left(u_{t}\right)=0 \text { in } \Omega \times(0,+\infty),
$$

where $\Omega$ is a bounded domain of $\mathbb{R}^{n}$ with smooth boundary $\partial \Omega$. They considered the well-known multiplier given by the vector field $m(x):=x-x^{0}, x^{0} \in \mathbb{R}^{n}$ arbitrarily chosen, but fixed, taken out of the domain $\Omega$, according to Figure 1 .

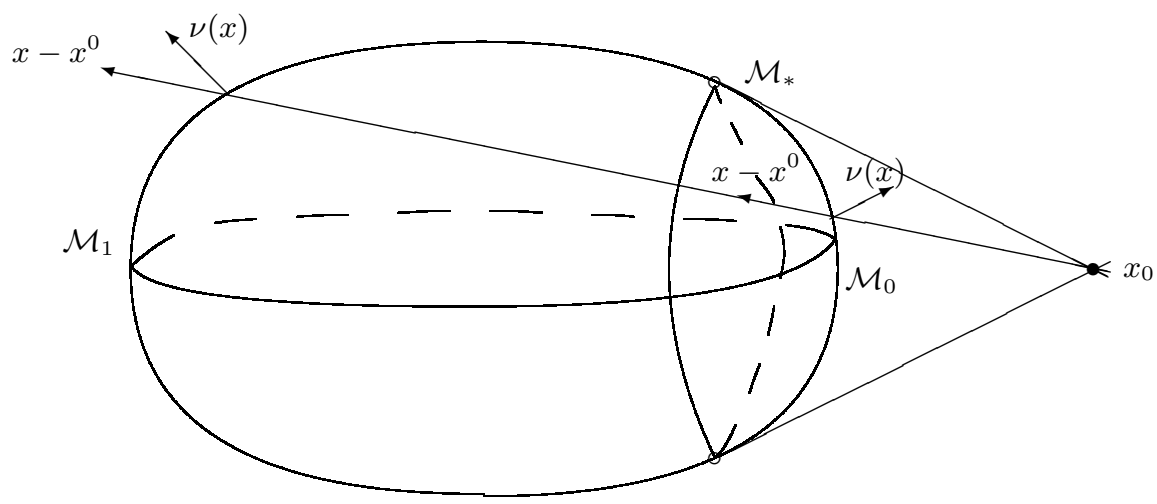

Figure 1. The observer is at $x_{0}$. The subset $\mathcal{M}_{0}$ is the "visible" part of $\mathcal{M}$ and $\mathcal{M}_{1}$ is its complement. The subset $\mathcal{M}_{*} \supset$ $\mathcal{M} \backslash \bigcup_{i=1}^{k} \mathcal{M}_{0 i}$ is an open set which contains $\mathcal{M} \backslash \bigcup_{i=1}^{k} \mathcal{M}_{0 i}$ and the damping is effective there. Observe that in Figure $1, k=1$ and $\mathcal{M}_{0 i}=\mathcal{M}_{01}=\mathcal{M}_{0}$. 
Once the multiplier $m(x)=x-x^{0}$ is not intrinsically connected with the manifold $\mathcal{M}$ they have to impose a restriction on the part $\mathcal{M}_{0}$ (without damping); namely, $\mathcal{M}_{0}$ must be umbilical, or umbilical by parts.

The main goal of the present manuscript is to improve considerably the previous result due to [Ca-Do-Fu-So, reducing arbitrarily the volume of the region where the dissipative effect lies. For this purpose we will construct an intrinsic multiplier that will play a crucial role when establishing the desired uniform decay rates of the energy. Fix $\epsilon>0$. This multiplier is, roughly speaking, given by the $\nabla_{T} f$, where $f: \mathcal{M} \rightarrow \mathbb{R}$ is a regular function which satisfies Hessf $\approx \mathbf{g}$ and $\inf _{x \in V}|\nabla f(x)|>0$ on a subset $V$ of $\mathcal{M}$ such that meas $(V) \geq \operatorname{meas}(\mathcal{M})-\epsilon$. This construction will be clarified in subsections 4.3 and 4.4 .

We would like to emphasize that the proofs of [Ra-Ta, BAR, HIT, based on microlocal analysis, do not extend to the nonlinear problem (1.1). In addition, making use of arguments due to Cavalcanti, Domingos Cavalcanti and Lasiecka CA-DO-LA, we obtain explicit and optimal decay rates of the energy. The decay rates obtained are optimal, since they are the same as the optimal rates derived in the works of Alabau-Boussouira ALA or Toudykov Tou.

Our paper is organized as follows. Section 2 is concerned with the statement of the problem and we introduce some notation. Our main result is stated in Section 3. Section 4 is devoted to the proof of the main result.

\section{Statement of the Problem}

Let $\mathcal{M}$ be a smooth oriented embedded compact surface without boundary in $\mathbb{R}^{3}$. For $\epsilon>0$ we prove that there exist an open subset $V \subset \mathcal{M}$ and a smooth function $f: \mathcal{M} \rightarrow \mathbb{R}$ such that meas $(V) \geq$ meas $(\mathcal{M})-\epsilon$,Hessf $\approx \mathbf{g}$ on $V$ and $\inf _{x \in V}|\nabla f(x)|>0$ (see Subsection 4.4).

In this paper, we investigate the stability properties of the function $u(x, t)$ which solves the damped problem

$$
\left\{\begin{array}{l}
\left.u_{t t}-\Delta_{\mathcal{M}} u+a(x) g\left(u_{t}\right)=0 \quad \text { on } \mathcal{M} \times\right] 0, \infty[, \\
u(0)=u^{0}, \quad u_{t}(0)=u^{1},
\end{array}\right.
$$

where the feedback function $g$ satisfies the Assumption 2.1.

\section{Assumption 2.1.}

(i) $g(s)$ is continuous and monotone increasing,

(ii) $g(s) s>0$ for $s \neq 0$,

(iii) $k|s| \leq g(s) \leq K|s|$ for $|s|>1$,

where $k$ and $K$ are two positive constants.

In addition, to obtain the stabilization of problem (2.1), we shall need the following geometrical assumption:

Assumption 2.2. Assume that $a \in L^{\infty}(\mathcal{M})$ is a nonnegative function such that

$$
a(x) \geq a_{0}>0, \quad \text { a.e. on } \mathcal{M}_{*},
$$

where $\mathcal{M}_{*}$ is an open set of $\mathcal{M}$ which contains $\mathcal{M} \backslash V$.

In the sequel, we are going to consider $\Sigma=\mathcal{M} \times] 0, T[$ and the Sobolev spaces $H^{s}(\mathcal{M}), s \in \mathbb{R}$, as in Lions and Magenes [LiMa, section 7.3]. 
On the other hand, using the Laplace-Beltrami operator $\Delta_{\mathcal{M}}$ on $\mathcal{M}$, we can give a more intrinsic definition of the spaces $H^{s}(\mathcal{M})$. Consider

$$
H^{2 m}(\mathcal{M})=\left\{u \in L^{2}(\mathcal{M}) / \Delta_{\mathcal{M}}^{m} u \in L^{2}(\mathcal{M})\right\},
$$

which, equipped with the canonical norm

$$
\|u\|_{H^{2 m}(\mathcal{M})}^{2}=\|u\|_{L^{2}(\mathcal{M})}^{2}+\left\|\Delta_{\mathcal{M}}^{m} u\right\|_{L^{2}(\mathcal{M})}^{2},
$$

is a Hilbert space.

We set

$$
V:=\left\{v \in H^{1}(\mathcal{M}) ; \int_{\mathcal{M}} v(x) d \mathcal{M}=0\right\},
$$

which is a Hilbert space with the topology endowed by $H^{1}(\mathcal{M})$.

Note that the condition $\int_{\mathcal{M}} v(x) d \mathcal{M}=0$ is required in order to guarantee the validity of the Poincaré inequality,

$$
\|f\|_{L^{2}(\mathcal{M})}^{2} \leq\left(\lambda_{1}\right)^{-1}\left\|\nabla_{T} f\right\|_{L^{2}(\mathcal{M})}^{2}, \quad \text { for all } f \in V,
$$

where $\lambda_{1}$ is the first eigenvalue of the Laplace-Beltrami operator.

Remark 2.1. It is convenient to observe that the space $V$ may not be invariant under the flow because of the nonlinear character of the equation under consideration. In this case, it is sufficient to add an extra term $\alpha u(\alpha>0)$ in the equation in order to control $L_{2}$ norms. However, for simplicity in the computations, we shall omit this term since it does not bring any additional difficulty or novelty.

We observe that problem (2.1) can be rewritten as

$$
\frac{d U}{d t}+\mathcal{A} U=G(U)
$$

where

$$
\mathcal{A}=\left(\begin{array}{cc}
0 & -I \\
-\Delta_{\mathcal{M}} & 0
\end{array}\right)
$$

is a maximal monotone operator and $G(\cdot)$ represents a locally Lipschitz perturbation. So, making use of standard semigroup arguments we have the following result:

\section{Theorem 2.1.}

- (i) Under the above-mentioned conditions, problem (2.1) is well-posed in the space $V \times L^{2}(\mathcal{M})$; that is, for any initial data $\left\{u^{0}, u^{1}\right\} \in V \times L^{2}(\mathcal{M})$, there exists a unique weak solution of (2.1) in the class

$$
u \in C\left(\mathbb{R}_{+} ; V\right) \cap C^{1}\left(\mathbb{R}_{+} ; L^{2}(\mathcal{M})\right) .
$$

- (ii) In addition, the velocity of the solution has the regularity given by

$$
u_{t} \in L_{l o c}^{2}\left(\mathbb{R}_{+} ; L^{2}(\mathcal{M})\right),
$$

and, consequently, $g\left(u_{t}\right) \in L_{\text {loc }}^{2}\left(\mathbb{R}_{+} ; L^{2}(\mathcal{M})\right)$ by Assumption 2.1 .

Furthermore, if $\left\{u^{0}, u^{1}\right\} \in V \cap H^{2}(\mathcal{M}) \times V$, then the solution has the following regularity:

$$
u \in L^{\infty}\left(\mathbb{R}_{+} ; V \cap H^{2}(\mathcal{M})\right) \cap W^{1, \infty}\left(\mathbb{R}_{+} ; V\right) \cap W^{2, \infty}\left(\mathbb{R}_{+} ; L^{2}(\mathcal{M})\right) .
$$


Consider that $u$ is the unique global weak solution of problem (2.1) given by Theorem 2.1. We define the corresponding energy functional by

$$
E(t)=\frac{1}{2} \int_{\mathcal{M}}\left[\left|u_{t}(x, t)\right|^{2}+\left|\nabla_{T} u(x, t)\right|^{2}\right] d \mathcal{M}
$$

For every solution of (2.1), in the class (2.5) we obtain for all $t_{2}>t_{1} \geq 0$,

$$
E\left(t_{2}\right)-E\left(t_{1}\right)=-\int_{t_{1}}^{t_{2}} \int_{\mathcal{M}} a(x) g\left(u_{t}\right) u_{t} d \mathcal{M} d t
$$

and, therefore, the energy is a nonincreasing function of the time variable $t$.

\section{Main Result}

In order to state the stability result, we need to define some functions which were first introduced in Lasiecka and Tataru LA-TA. For the reader's comprehension we will repeat them briefly. Let $h$ be a concave, strictly increasing function, with $h(0)=0$, and such that

$$
h(s g(s)) \geq s^{2}+g^{2}(s), \text { for }|s| \leq 1 .
$$

Note that such a function can be straightforwardly constructed, considering the hypotheses on $g$ in Assumption 2.1. In view of this function, we define

$$
r(.)=h\left(\frac{\cdot}{\operatorname{meas}\left(\Sigma_{1}\right)}\right) .
$$

As $r$ is monotone increasing, then $c I+r$ is invertible for all $c \geq 0$. For $L$ a positive constant, we set

$$
p(x)=(c I+r)^{-1}(L x),
$$

where the function $p$ is easily seen to be positive, continuous and strictly increasing with $p(0)=0$. Finally, let

$$
q(x)=x-(I+p)^{-1}(x) .
$$

We can now proceed to state our stability result.

Theorem 3.1. Assume that Assumption 2.1 and Assumption 2.2 are in place. Let $u$ be the weak solution of problem (2.1). With the energy $E(t)$ defined as in (2.7), there exists $T_{0}>0$ such that

$$
E(t) \leq S\left(\frac{t}{T_{0}}-1\right), \quad \forall t>T_{0},
$$

with $\lim _{t \rightarrow \infty} S(t)=0$, where the contraction semigroup $S(t)$ is the solution of the differential equation

$$
\frac{d}{d t} S(t)+q(S(t))=0, \quad S(0)=E(0)
$$

where $q$ is given in (3.4), the constant $L$, which is given in (3.3), depends on meas $(\Sigma)$ and the constant $c$ is equal to $\frac{k^{-1}+K}{\text { meas }(\Sigma)\left(1+\|a\|_{\infty}\right)}$.

Remark 3.1. If the feedback is linear, e.g., $g(s)=s$, then, under the same assumptions of Theorem 3.1, we obtain that the energy of problem (2.1) decays exponentially with respect to the initial energy; that is, there exist two positive constants $C>0$ and $k>0$ such that

$$
E(t) \leq C e^{-k t} E(0), \quad t>0
$$


If we consider $g(s)=s^{p}, p>1$ at the origin, and since the function $s^{\frac{p+1}{2}}$ is convex for $p>1$, then, solving

$$
S_{t}+S^{\frac{p+1}{2}}=0
$$

we obtain the following polynomial decay rate:

$$
E(t) \leq C(E(0))\left[E(0)^{\frac{-p+1}{2}}+t(p-1)\right]^{\frac{2}{-p+1}} .
$$

We can find more examples of explicit decay rates in Cavalcanti, Domingos Cavalcanti and Lasiecka [CA-DO-LA].

\section{Proof of MAIN Result}

\subsection{Preliminaries.}

We collect, below, a few formulas to be invoked in the sequel.

Let $\nu$ be the exterior normal vector field on $\mathcal{M}$. The Laplace-Beltrami operator $\Delta_{\mathcal{M}}$ of a function $\varphi: \mathcal{M} \rightarrow \mathbb{R}$ of class $C^{2}$ is defined by

$$
\Delta_{\mathcal{M}} \varphi:=\operatorname{div}_{T} \nabla_{T} \varphi
$$

where $\operatorname{div}_{T} \nabla_{T} \varphi$, is the divergent of the vector field $\nabla_{T} \varphi$.

Assuming that $\varphi: \mathcal{M} \rightarrow \mathbb{R}$ is a function of class $C^{1}$ and $x \in \mathcal{M} \mapsto q(x) \in T_{x}(\mathcal{M})$ is a vector field of class $C^{1}$, we have

$$
\begin{aligned}
\int_{\mathcal{M}} q \cdot \nabla_{T} \varphi d \mathcal{M} & =-\int_{\mathcal{M}} \operatorname{div}_{T} q \varphi d \mathcal{M}, \\
2 \varphi\left(q \cdot \nabla_{T} \varphi\right) & =q \cdot \nabla_{T}\left(\varphi^{2}\right) .
\end{aligned}
$$

From (4.2) and (4.3), we conclude the following formula:

$$
2 \int_{\mathcal{M}} \varphi\left(q \cdot \nabla_{T} \varphi\right) d \mathcal{M}=\int_{\mathcal{M}} q \cdot \nabla_{T}\left(\varphi^{2}\right) d \mathcal{M}=-\int_{\mathcal{M}} \operatorname{div}_{T} q|\varphi|^{2} d \mathcal{M}
$$

We define a continuous linear operator $-\Delta_{\tilde{\mathcal{M}}}: H^{1}(\tilde{\mathcal{M}}) \rightarrow\left(H^{1}(\tilde{\mathcal{M}})\right)^{\prime}$, where $\tilde{\mathcal{M}}$ is a nonempty open subset of $\mathcal{M}$ (sometimes the whole $\mathcal{M}$ ) such that

$$
\left\langle-\Delta_{\tilde{\mathcal{M}}} \varphi, \psi\right\rangle=\int_{\tilde{\mathcal{M}}} \nabla_{T} \varphi \cdot \nabla_{T} \psi d \mathcal{M}, \quad \forall \varphi, \psi \in H^{1}(\tilde{\mathcal{M}})
$$

and, in particular,

$$
\left\langle-\Delta_{\tilde{\mathcal{M}}} \varphi, \varphi\right\rangle=\int_{\tilde{\mathcal{M}}}\left|\nabla_{T} \varphi\right|^{2} d \mathcal{M}, \quad \forall \varphi \in H^{1}(\tilde{\mathcal{M}}) .
$$

The operator $-\Delta_{\tilde{\mathcal{M}}}+I$ defines an isomorphism from $H^{1}(\tilde{\mathcal{M}})$ over $\left[H^{1}(\tilde{\mathcal{M}})\right]^{\prime}$. We observe that when $\tilde{\mathcal{M}}$ is a manifold without boundary, and this is the case, for instance, if $\tilde{\mathcal{M}}=\mathcal{M}$, we have $H^{1}(\tilde{\mathcal{M}})=H_{0}^{1}(\tilde{\mathcal{M}})$ and, consequently, $\left[H^{1}(\tilde{\mathcal{M}})\right]^{\prime}=$ $H^{-1}(\tilde{\mathcal{M}})$.

Remark 4.1. It is convenient to observe that all the above classical formulas can be extended to Sobolev spaces using density arguments.

The proof of Theorem 3.1 proceeds through several steps. In order to obtain the decay rate stated in (3.5), we will consider, initially, regular solutions of problem (2.1). Then, making use of standard density arguments, the estimate (3.5) holds for weak solutions. 
4.2. An identity. We begin by proving the following proposition:

Proposition 4.2.1. Let $\mathcal{M} \subset \mathbb{R}^{3}$ be an oriented regular compact surface without boundary and $q$ a vector field of class $C^{1}$. Then, for every regular solution $u$ of (1.1) we have the following identity:

$$
\begin{aligned}
& {\left[\int_{\mathcal{M}} u_{t} q \cdot \nabla_{T} u d \mathcal{M}\right]_{0}^{T}+\frac{1}{2} \int_{0}^{T} \int_{\mathcal{M}}\left(\operatorname{div}_{T} q\right)\left\{\left|u_{t}\right|^{2}-\left|\nabla_{T} u\right|^{2}\right\} d \mathcal{M} d t} \\
& +\int_{0}^{T} \int_{\mathcal{M}} \nabla_{T} u \cdot \nabla_{T} q \cdot \nabla_{T} u d \mathcal{M} d t+\int_{0}^{T} \int_{\mathcal{M}} a(x) g\left(u_{t}\right)\left(q \cdot \nabla_{T} u\right) d \mathcal{M} d t=0 .
\end{aligned}
$$

Proof. Multiplying the equation (1.1) by the multiplier $q \cdot \nabla_{T} u$ and integrating on $\mathcal{M} \times] 0, T[$, we obtain

$$
0=\int_{0}^{T} \int_{\mathcal{M}}\left(u_{t t}-\Delta_{\mathcal{M}} u+a(x) g\left(u_{t}\right)\right)\left(q \cdot \nabla_{T} u\right) d \mathcal{M} d t
$$

Next, we will estimate some terms on the RHS of identity (4.8). Taking (4.2), (4.3) and (4.4) into account, we obtain

$$
\begin{aligned}
& \int_{0}^{T} \int_{\mathcal{M}}\left(-\Delta_{\mathcal{M}} u\right)\left(q \cdot \nabla_{T} u\right) d \mathcal{M} d t=\int_{0}^{T} \int_{\mathcal{M}} \nabla_{T} u \cdot \nabla_{T}\left(q \cdot \nabla_{T} u\right) d \mathcal{M} d t \\
& =\int_{0}^{T} \int_{\mathcal{M}} \nabla_{T} u \cdot \nabla_{T} q \cdot \nabla_{T} u d \mathcal{M} d t+\frac{1}{2} \int_{0}^{T} \int_{\mathcal{M}} q \cdot \nabla_{T}\left[\left|\nabla_{T} u\right|^{2}\right] d \mathcal{M} d t \\
& =\int_{0}^{T} \int_{\mathcal{M}} \nabla_{T} u \cdot \nabla_{T} q \cdot \nabla_{T} u d \mathcal{M} d t-\frac{1}{2} \int_{0}^{T} \int_{\mathcal{M}}\left|\nabla_{T} u\right|^{2} d i v_{T} q d \mathcal{M} d t
\end{aligned}
$$

and, integrating by parts and considering (4.4), we obtain

$$
\begin{aligned}
& \int_{0}^{T} \int_{\mathcal{M}}\left(u_{t t}+a(x) g\left(u_{t}\right)\right)\left(q \cdot \nabla_{T} u\right) d \mathcal{M} d t \\
= & {\left[\int_{\mathcal{M}} u_{t}\left(q \cdot \nabla_{T} u\right)\right]_{0}^{T}-\int_{0}^{T} \int_{\mathcal{M}} u_{t}\left(q \cdot \nabla_{T} u_{t}\right) d \mathcal{M} d t } \\
& +\int_{0}^{T} \int_{\mathcal{M}} a(x) g\left(u_{t}\right)\left(q \cdot \nabla_{T} u\right) d \mathcal{M} d t \\
= & {\left[\int_{\mathcal{M}} u_{t}\left(q \cdot \nabla_{T} u\right)\right]_{0}^{T}+\frac{1}{2} \int_{0}^{T} \int_{\mathcal{M}}\left(d i v_{T} q_{T}\right)\left|u_{t}\right|^{2} d \mathcal{M} d t } \\
& +\int_{0}^{T} \int_{\mathcal{M}} a(x) g\left(u_{t}\right)\left(q \cdot \nabla_{T} u\right) d \mathcal{M} d t .
\end{aligned}
$$

Combining (4.8), (4.9) and (4.10), we deduce (4.7), which concludes the proof of Proposition 4.2.1. 
Employing (4.7) with $q(x)=\nabla_{T} f$ where $f: \mathcal{M} \rightarrow \mathbb{R}$ is a $C^{3}$ function to be determined later, we infer

$$
\begin{aligned}
& {\left[\int_{\mathcal{M}} u_{t} \nabla_{T} f \cdot \nabla_{T} u d \mathcal{M}\right]_{0}^{T}+\frac{1}{2} \int_{0}^{T} \int_{\mathcal{M}} \Delta_{\mathcal{M}} f\left\{\left|u_{t}\right|^{2}-\left|\nabla_{T} u\right|^{2}\right\} d \mathcal{M} d t} \\
& +\int_{0}^{T} \int_{\mathcal{M}}\left(\nabla_{T} u \cdot \operatorname{Hess}(f) \cdot \nabla_{T} u\right) d \mathcal{M} d t \\
& +\int_{0}^{T} \int_{\mathcal{M}} a(x) g\left(u_{t}\right)\left(\nabla_{T} f \cdot \nabla_{T} u\right) d \mathcal{M} d t=0 .
\end{aligned}
$$

We have the following identity:

Lemma 4.2.3. Let $u$ be a weak solution to problem (1.1) and let $\xi \in C^{1}(\mathcal{M})$. Then

$$
\begin{aligned}
{\left[\int_{\mathcal{M}} u_{t} \xi u d \mathcal{M}\right]_{0}^{T} } & =\int_{0}^{T} \int_{\mathcal{M}} \xi\left|u_{t}\right|^{2} d \mathcal{M} d t-\int_{0}^{T} \int_{\mathcal{M}} \xi\left|\nabla_{T} u\right|^{2} d \mathcal{M} d t \\
& -\int_{0}^{T} \int_{\mathcal{M}}\left(\nabla_{T} u \cdot \nabla_{T} \xi\right) u d \mathcal{M} d t-\int_{0}^{T} \int_{\mathcal{M}} a(x) g\left(u_{t}\right) \xi u d \mathcal{M} d t .
\end{aligned}
$$

Proof. Multiplying the equation of (1.1) by $\xi u$ and integrating by parts we obtain the desired result.

Substituting $\xi=\alpha>0$ in (4.12) and combining the obtained result with identity (4.11) we deduce

$$
\begin{aligned}
& \int_{0}^{T} \int_{\mathcal{M}}\left(\frac{\Delta_{\mathcal{M}} f}{2}-\alpha\right)\left|u_{t}\right|^{2} d \mathcal{M} d t \\
& +\int_{0}^{T} \int_{\mathcal{M}}\left[\left(\nabla_{T} u \cdot \operatorname{Hess}(f) \cdot \nabla_{T} u\right)+\left(\alpha-\frac{\Delta_{\mathcal{M}} f}{2}\right)\left|\nabla_{T} u\right|^{2}\right] d \mathcal{M} d t \\
& =-\left[\int_{\mathcal{M}} u_{t} \nabla_{T} f \cdot \nabla_{T} u d \mathcal{M}\right]_{0}^{T}-\alpha\left[\int_{\mathcal{M}} u_{t} u d \mathcal{M}\right]_{0}^{T} \\
& -\alpha \int_{0}^{T} \int_{\mathcal{M}} a(x) g\left(u_{t}\right) u d \mathcal{M} d t \\
& -\int_{0}^{T} \int_{\mathcal{M}} a(x) g\left(u_{t}\right)\left(\nabla_{T} f \cdot \nabla_{T} u\right) d \mathcal{M} d t
\end{aligned}
$$

Remark 4.2. This is the precise moment where the properties of the function $f$ play an important role. Note that what we just need is to find a subset $V$ of $\mathcal{M}$ such that

$$
\begin{aligned}
& C \int_{0}^{T} \int_{V}\left[u_{t}^{2}+\left|\nabla_{T} u\right|^{2}\right] d \mathcal{M} d t \\
& \leq \int_{0}^{T} \int_{V}\left(\frac{\Delta_{\mathcal{M}} f}{2}-\alpha\right)\left|u_{t}\right|^{2} d \mathcal{M} d t \\
& +\int_{0}^{T} \int_{V}\left[\left(\nabla_{T} u \cdot \operatorname{Hess}(f) \cdot \nabla_{T} u\right)+\left(\alpha-\frac{\Delta_{\mathcal{M}} f}{2}\right)\left|\nabla_{T} u\right|^{2}\right] d \mathcal{M} d t
\end{aligned}
$$


for some positive constant $C$, provided that $\alpha$ is suitably chosen. Assuming, for a moment, that (4.14) holds, (4.13) yields

$$
\begin{aligned}
2 C \int_{0}^{T} E(t) d t & \leq C \int_{0}^{T} \int_{\mathcal{M} \backslash V}\left[u_{t}^{2}+\left|\nabla_{T} u\right|^{2}\right] d \mathcal{M} d t \\
& +\left|\left[\int_{\mathcal{M}} u_{t} \nabla_{T} f \cdot \nabla_{T} u d \mathcal{M}\right]_{0}^{T}\right|+\alpha\left|\left[\int_{\mathcal{M}} u_{t} u d \mathcal{M}\right]_{0}^{T}\right| \\
& +\left|\alpha \int_{0}^{T} \int_{\mathcal{M}} a(x) g\left(u_{t}\right) u d \mathcal{M} d t\right| \\
& +\left|\int_{0}^{T} \int_{\mathcal{M}} a(x) g\left(u_{t}\right)\left(\nabla_{T} f \cdot \nabla_{T} u\right) d \mathcal{M} d t\right| .
\end{aligned}
$$

The inequality (4.15) is controlled by considering a standard procedure, which, for the reader's convenience, we will repeat later. The main idea behind this procedure is to consider the dissipative area, namely, $\mathcal{M}_{*}$, containing the set $\mathcal{M} \backslash V$ as stated in (2.2). It is important to observe that $\mathcal{M}_{*}$ is as small as $V$ can be big.

The next subsections are devoted to the construction of a function $f$ as well as a subset $V$ of $\mathcal{M}$ such that the inequality (4.14) holds. This will be done, for simplicity, in a general setting, that is, for a Riemannian manifold (without boundary) with Riemmanian metric $\mathbf{g}$ of class $C^{2}$.

\subsection{Construction of a function such that Hessf $\approx \mathbf{g}$ and $\inf _{x \in V}|\nabla f(x)|>0$ locally.}

Throughout this subsection we are going to denote the Laplacian-Beltrami operator $\Delta_{\mathcal{M}}$ by $\Delta$ and the tangential-gradient $\nabla_{T}$ by $\nabla$. Let $\mathcal{M}$ be a compact $n$-dimensional Riemannian manifold (without boundary) with Riemmanian metric $\mathrm{g}$ of class $C^{2}$. Let $\nabla$ denote the Levi-Civita connection. Fix $p \in \mathcal{M}$. Our aim is to construct a function $f: V_{p} \rightarrow \mathbb{R}$ such that Hessf $\approx \mathrm{g}$ and $\inf _{x \in V_{p}}|\nabla f(x)|>0$, where $V_{p}$ is a neighborhood of $p$ and the Hessian of $f$ is seen as a bilinear form defined on the tangent space $T_{p} \mathcal{M}$ of $\mathcal{M}$ at $p$.

We begin with an orthonormal basis $\left(e_{1}, \ldots, e_{n}\right)$ of $T_{p} M$. Define a normal coordinate system $\left(x_{1}, \ldots, x_{n}\right)$ in a neighborhood $\widetilde{V}_{p}$ of $p$ such that $\partial / \partial x_{i}(p)=e_{i}(p)$ for every $i=1, \ldots, n$. It is well known that in this coordinate system we have that $\Gamma_{i j}^{k}(p)=0$, where $\Gamma_{i j}^{k}$ are the Christoffel symbols with respect to $\left(x_{1}, \ldots, x_{n}\right)$ (see, for instance, Do Carmo).

The Hessian with respect to $\left(x_{1}, \ldots, x_{n}\right)$ is given by

$$
\operatorname{Hessf}\left(\frac{\partial}{\partial x_{i}}, \frac{\partial}{\partial x_{j}}\right)=\frac{\partial^{2} f}{\partial x_{i} \partial x_{j}}-\sum_{k=1}^{n} \Gamma_{i j}^{k} \frac{\partial f}{\partial x_{k}} \text {. }
$$

The Laplacian of $f$ is the trace of the Hessian with respect to the metric $\mathbf{g}$. If $\mathbf{g}_{i j}$ denote the components of the Riemannian metric with respect to $\left(x_{1}, \ldots, x_{n}\right)$ and $\mathbf{g}^{i j}$ are the components of the inverse matrix of $\mathbf{g}_{i j}$, then the Laplacian of $f$ is given by

$$
\Delta f=\sum_{i, j} \mathbf{g}^{i j} H \operatorname{essf}\left(\frac{\partial}{\partial x_{i}}, \frac{\partial}{\partial x_{j}}\right)
$$


Consider the function $f: \widetilde{V}_{p} \rightarrow \mathbb{R}$ defined by

$$
f(x)=x_{1}+\frac{1}{2} \sum_{i=1}^{n} x_{i}^{2}
$$

It is immediate that $\Delta f(p)=n$ and $|\nabla f(p)|=1$. Moreover, Hessf $(p)=\mathbf{g}(p)$, which implies that

$$
\operatorname{Hessf}(p)(v, v)=|v|_{p}^{2}
$$

We are interested in finding a neighborhood $V_{p} \subset \widetilde{V}_{p}$ of $p$ and a strictly positive constant $C$ such that

$$
\begin{aligned}
C \int_{0}^{T} & \int_{V_{p}}\left(|\nabla u|^{2}+u_{t}^{2}\right) d \mathcal{M} d t \\
& \leq \int_{0}^{T} \int_{V_{p}}\left[H \operatorname{essf}(\nabla u, \nabla u)+\left(\alpha-\frac{\Delta f}{2}\right)|\nabla u|^{2}+\left(\frac{\Delta f}{2}-\alpha\right) u_{t}^{2}\right] d \mathcal{M} d t,
\end{aligned}
$$

for some $\alpha \in \mathbb{R}$. We claim that if we consider $\alpha=\frac{n}{2}-\frac{1}{2}$ and $C=1 / 4$ we obtain the desired inequality, which means that it is enough to prove that there exist $V_{p} \subset \widetilde{V}_{p}$ satisfying

$$
\int_{0}^{T} \int_{V_{p}} \operatorname{Hessf}(\nabla u, \nabla u)+\left(\frac{n}{2}-\frac{3}{4}-\frac{\Delta f}{2}\right)|\nabla u|^{2} d \mathcal{M} d t \geq 0
$$

and

$$
\int_{0}^{T} \int_{V_{p}}\left(\frac{\Delta f}{2}-\frac{n}{2}+\frac{1}{4}\right) u_{t}^{2} d \mathcal{M} d t \geq 0
$$

In order to prove the existence of a subset $V_{p} \subset \widetilde{V}_{p}$ where (4.17) holds, let $\theta_{1}$ be the smooth field of symmetric bilinear forms on $\widetilde{V}_{p}$ defined as

$$
\theta_{1}(X, Y)=\operatorname{Hessf}(X, Y)+\left(\frac{n}{2}-\frac{3}{4}-\frac{\Delta f}{2}\right) \mathbf{g}(X, Y)
$$

where $X$ and $Y$ are vector fields on $\widetilde{V}_{p}$. It is clearly a positive definite bilinear form on $p$ since $\operatorname{Hessf}(p)(X, Y)=\mathbf{g}(p)(X, Y)$ and

$$
\theta_{1}(p)(X, Y)=\frac{1}{4} \mathbf{g}(p)(X, Y) .
$$

Therefore, there exists a neighborhood $\widehat{V}_{p}$ such that $\theta_{1}$ is positive definite and

$$
\int_{0}^{T} \int_{\widehat{V}_{p}} \operatorname{Hessf}(\nabla u, \nabla u)+\left(\frac{n}{2}-\frac{3}{4}-\frac{\Delta f}{2}\right)|\nabla u|^{2} d \mathcal{M} d t \geq 0 .
$$

To prove the existence of $\breve{V}_{p} \subset \widetilde{V}_{p}$ such that (4.18) holds is easier. It is enough to notice that at $p$ we have that

$$
\left(\frac{\Delta f(p)}{2}-\frac{n}{2}+\frac{1}{4}\right)=\frac{1}{4}
$$


and the existence of $\breve{V}_{p} \subset \widetilde{V}_{p}$ such that (4.18) holds is immediate. Furthermore we can eventually choose a smaller $V_{p}$ such that $\inf _{x \in V_{p}}|\nabla f(x)|>0$. Therefore the existence of $V_{p} \subset \widetilde{V}_{p}$ such that $\inf _{x \in V_{p}}|\nabla f(x)|>0$ and (4.16) holds is settled.

4.4. A function $f$ that satisfies inequality (4.16) and $\inf _{x \in V}|\nabla f(x)|>0$ in a wide domain.

In what follows, $\bar{V}$ denotes the closure of $V$ and $\partial V$ denotes the boundary of $V$. When $\bar{V} \subset W$ is bounded, we say that $V$ is compactly contained in $W$ and we denote this relationship by $V \Subset W$.

Theorem 4.1. Let $(\mathcal{M}, \mathbf{g})$ be a two-dimensional Riemannian manifold. Then, for every $\epsilon>0$, there exist a finite family $\left\{V_{i}\right\}_{i=1 \ldots k}$ of open sets with smooth boundary, smooth functions $f_{i}: \bar{V}_{i} \rightarrow \mathbb{R}$ and a constant $C>0$ such that

(1) the subsets $\bar{V}_{i}$ are pairwise disjoint;

(2) $\operatorname{vol}\left(\bigcup_{i=1}^{k} V_{i}\right) \geq \operatorname{vol}(M)-\epsilon$;

(3) inequality (4.16) holds for every $f_{i}$;

(4) $\inf _{x \in V_{i}}|\nabla f(x)|>0$ for every $i=1, \ldots, k$.

Proof. First of all, it is possible to get open subsets $\left\{\widetilde{W}_{j}\right\}_{j=1, \ldots, s}$ with smooth boundaries and a family of smooth functions $\left\{\widetilde{f}_{j}: \widetilde{W}_{j} \rightarrow \mathbb{R}\right\}_{j=1, \ldots, s}$ such that $\left\{\widetilde{W}_{j}\right\}_{j=1, \ldots, s}$ is a cover of $\mathcal{M}$ and each $\widetilde{f}_{j}$ satisfies inequality (4.16). Moreover, we can choose $\widetilde{W}_{j}$ in such a way that their boundaries intercept themselves transversally and three or more boundaries do not intercept themselves at the same point.

Set $A:=\bigcup_{j=1}^{s} \partial \widetilde{W}_{j}$. Then, $\mathcal{M} \backslash A$ is a disjoint union of connected open sets $\bigcup_{i=1}^{k} W_{i}$ such that $\partial W_{i}$ is a piecewise smooth curve.

Each $W_{i}$ is contained in some $\widetilde{W}_{j}$. Therefore, for each $W_{i}$, choose a function $\hat{f}_{i}:=\left.\widetilde{f}_{j}\right|_{W_{i}}$.

The open subsets $V_{i}, i=1, \ldots, k$, we are looking for are subsets of $W_{i}$. We can choose them in such a way that

(1) $V_{i} \Subset W_{i}$;

(2) $\partial V_{i}$ is smooth;

(3) $\operatorname{vol}\left(W_{i}\right)-\operatorname{vol}\left(V_{i}\right)<\epsilon / k$.

Finally, if we set $f_{i}=\left.\hat{f}_{i}\right|_{\bar{V}_{i}}$, we prove the theorem.

Theorem 4.2. Let $(\mathcal{M}, \mathbf{g})$ be a two-dimensional Riemannian manifold. Fix $\epsilon>0$. Then, there exists a smooth function $f: M \rightarrow \mathbb{R}$ such that inequality (4.16) and the condition $\inf _{x \in V}|\nabla f(x)|>0$ hold in a subset $V$ with $\operatorname{vol}(V) \geq \operatorname{vol}(\mathcal{M})-\epsilon$.

Proof. Consider Theorem 4.1 and the constructions made in its proof. Denote $\lambda:=\min _{i \neq j} \operatorname{dist}\left(V_{i}, V_{j}\right)>0$. Consider a tubular neighborhood $V^{\delta}$ of $V=\bigcup_{i=1}^{k} V_{i}$ of the points whose distance is less than or equal to $\delta<\lambda / 4$. Then, it is possible to define a smooth (cut-off) function $\eta: \mathcal{M} \rightarrow \mathbb{R}$ such that

$$
\eta(x)=\left\{\begin{array}{l}
1 \text { if } x \in V \\
0 \text { if } x \in \mathcal{M} \backslash V^{\delta} \\
\text { between } 0 \text { and } 1 \text { otherwise. }
\end{array}\right.
$$


Now, notice that $f: \mathcal{M} \rightarrow \mathbb{R}$ defined by

$$
f(x)=\left\{\begin{array}{l}
\hat{f}_{i}(x) \eta(x) \text { if } x \in W_{i} \\
0 \text { otherwise }
\end{array}\right.
$$

is smooth and satisfies inequality (4.16) and the condition $\inf _{x \in V}|\nabla f(x)|>0$. In addition, the inequality $\operatorname{vol}(V) \geq \operatorname{vol}(\mathcal{M})-\epsilon$ holds, which settles the theorem.

We denote

$$
\chi=\left[\int_{\mathcal{M}} u_{t} \nabla_{T} f \cdot \nabla_{T} u d \mathcal{M}\right]_{0}^{T}+\alpha\left[\int_{\mathcal{M}} u_{t} u d \mathcal{M}\right]_{0}^{T} .
$$

Next we will estimate some terms in (4.15). Let us define

$$
R:=\max _{x \in \mathcal{M}}\left|\nabla_{T} f(x)\right| .
$$

Estimate for $I_{1}:=\int_{0}^{T} \int_{\mathcal{M}} a(x) g\left(u_{t}\right)\left(\nabla_{T} f \cdot \nabla_{T} u\right) d \mathcal{M} d t$.

By the Cauchy-Schwarz inequality, taking (4.20) into account and considering the inequality $a b \leq \frac{a^{2}}{4 \zeta}+\zeta b^{2}$, where $\zeta$ is a positive number, we obtain

$$
\left|I_{1}\right| \leq \frac{\|a\|_{L^{\infty}(\mathcal{M})} R^{2}}{\zeta} \int_{0}^{T} \int_{\mathcal{M}} a(x)\left|g\left(u_{t}\right)\right|^{2} d \mathcal{M} d t+2 \zeta \int_{0}^{T} E(t) d t
$$

Estimate for $I_{2}=\alpha \int_{0}^{T} \int_{\mathcal{M}} a(x) g\left(u_{t}\right) u d \mathcal{M} d t$.

Similarly we infer

$$
\left|I_{2}\right| \leq \frac{\|a\|_{L^{\infty}(\mathcal{M})} \alpha^{2} \lambda_{1}^{-1}}{16 \zeta} \int_{0}^{T} \int_{\mathcal{M}} a(x)\left|g\left(u_{t}\right)\right|^{2} d \mathcal{M} d t+2 \zeta \int_{0}^{T} E(t) d t,
$$

where $\lambda_{1}$ comes from the Poincaré inequality given in (2.4).

Choosing $\zeta$ sufficiently small and inserting (4.19), (4.21) and (4.22) into (4.15) yields

$$
\begin{aligned}
\int_{0}^{T} E(t) d t & \leq|\chi|+C_{1} \int_{0}^{T} \int_{\mathcal{M}} a(x)\left(g\left(u_{t}\right)\right)^{2} d \mathcal{M} d t \\
& +C_{1} \int_{0}^{T} \int_{\mathcal{M} \backslash V}\left[\left|\nabla_{T} u\right|^{2}+a(x) u_{t}^{2}\right] d \mathcal{M} d t
\end{aligned}
$$

where

$$
C_{1}:=C_{1}\left(C,\|a\|_{L^{\infty}(\mathcal{M})}, \lambda_{1}^{-1}, R, a_{0}^{-1}, n\right) .
$$

It remains to estimate the quantity $\int_{0}^{T} \int_{\mathcal{M} \backslash V}\left|\nabla_{T} u\right|^{2} d \mathcal{M} d t$ in terms of the damping term $\int_{0}^{T} \int_{\mathcal{M}}\left[a(x)\left|g\left(u_{t}\right)\right|^{2}+a(x)\left|u_{t}\right|^{2}\right] d \mathcal{M} d t$. For this purpose we have to build a "cut-off" function $\eta_{\varepsilon}$ on a specific neighborhood of $\mathcal{M} \backslash V$. First of all, define $\tilde{\eta}: \mathbb{R} \rightarrow \mathbb{R}$ such that

$$
\tilde{\eta}(x)=\left\{\begin{array}{ccc}
1 & \text { if } & x \leq 0 \\
(x-1)^{2} & \text { if } & x \in[1 / 2,1] \\
0 & \text { if } & x>1
\end{array}\right.
$$

and it is defined on $(0,1 / 2)$ in such a way that $\tilde{\eta}$ is a nondecreasing function of class $C^{1}$. For $\varepsilon>0$, set $\tilde{\eta}_{\varepsilon}(x):=\tilde{\eta}(x / \varepsilon)$. It is straightforward that there exists a 
constant $M$ which does not depend on $\varepsilon$ such that

$$
\frac{\left|\tilde{\eta}_{\varepsilon}^{\prime}(x)\right|^{2}}{\tilde{\eta}_{\varepsilon}(x)} \leq \frac{M}{\varepsilon^{2}}
$$

for every $x<\varepsilon$.

Let $\mathcal{M}_{*} \supset \mathcal{M} \backslash V$ be an open subset of $\mathcal{M}$ and let $\varepsilon>0$ be such that

$$
\tilde{\omega}_{\varepsilon}:=\{x \in \mathcal{M} ; \operatorname{dist}(x, \partial V)<\varepsilon\}
$$

is a tubular neighborhood of $\partial V$ and $\omega_{\varepsilon}:=\tilde{\omega}_{\varepsilon} \cup \mathcal{M} \backslash V$ is contained in $\mathcal{M}_{*}$. Define $\eta_{\varepsilon}: \mathcal{M} \rightarrow \mathbb{R}$ as

$$
\eta_{\varepsilon}(x)= \begin{cases}1 & \text { if } x \in \mathcal{M} \backslash V, \\ \tilde{\eta}_{\varepsilon}(d(x, \mathcal{M} \backslash V)) & \text { if } x \in \omega_{\varepsilon} \backslash(\mathcal{M} \backslash V), \\ 0 & \text { otherwise. }\end{cases}
$$

It is straightforward that $\eta_{\varepsilon}$ is a function of class $C^{1}$ on $\mathcal{M}$ due to the smoothness of $\partial(\mathcal{M} \backslash V)$ and $\partial \omega_{\varepsilon}$. Notice also that

$$
\frac{\left|\nabla_{T} \eta_{\varepsilon}(x)\right|^{2}}{\eta_{\varepsilon}(x)}=\frac{\left|\tilde{\eta}_{\varepsilon}^{\prime}\left(d\left(x, \mathcal{M}_{2}\right)\right)\right|^{2}}{\tilde{\eta}_{\varepsilon}\left(d\left(x, \mathcal{M}_{2}\right)\right)} \leq \frac{M}{\varepsilon^{2}}
$$

for every $x \in \omega_{\varepsilon}$. In particular, $\frac{\left|\nabla_{T} \eta_{\varepsilon}\right|^{2}}{\eta_{\varepsilon}} \in L^{\infty}\left(\omega_{\varepsilon}\right)$.

Taking $\xi=\eta_{\varepsilon}$ in the identity (4.12) we obtain

$$
\begin{aligned}
& \int_{0}^{T} \int_{\omega_{\varepsilon}} \eta_{\varepsilon}\left|\nabla_{T} u\right|^{2} d \mathcal{M} d t \\
= & -\left[\int_{\omega_{\varepsilon}} u_{t} u \eta_{\varepsilon} d \mathcal{M}\right]_{0}^{T}+\int_{0}^{T} \int_{\omega_{\varepsilon}} \eta_{\varepsilon}\left|u_{t}\right|^{2} d \mathcal{M} \\
- & \int_{0}^{T} \int_{\omega_{\varepsilon}} u\left(\nabla_{T} u \cdot \nabla_{T} \eta_{\varepsilon}\right) d \mathcal{M} d t-\int_{0}^{T} \int_{\omega_{\varepsilon}} a(x) g\left(u_{t}\right) u \eta_{\varepsilon} d \mathcal{M} d t .
\end{aligned}
$$

Next we will estimate the terms on the RHS of (4.25).

Estimate for $K_{1}:=\int_{0}^{T} \int_{\omega_{\varepsilon}} \eta_{\varepsilon}\left|u_{t}\right|^{2} d \mathcal{M} d t$.

From (2.2), since $\eta_{\varepsilon} \leq 1$ and $\omega_{\varepsilon} \subset \mathcal{M}_{*}$, where the damping lies, we deduce

$$
K_{1} \leq a_{0}^{-1} \int_{0}^{T} \int_{\mathcal{M}} a(x) u_{t}^{2} d \mathcal{M} d t
$$

Estimate for $K_{2}:=-\int_{0}^{T} \int_{\omega_{\varepsilon}} a(x) g\left(u_{t}\right) u \eta_{\varepsilon} d \mathcal{M} d t$.

Taking into account the Cauchy-Schwarz inequality, the inequality $a b \leq \frac{1}{4 \alpha} a^{2}+$ $\alpha b^{2}$ and (2.4) we obtain

$$
\left|K_{2}\right| \leq \frac{\lambda_{1}^{-1}|| a \|_{L^{\infty}(\mathcal{M})}}{4 \alpha} \int_{0}^{T} \int_{\mathcal{M}} a(x)\left|g\left(u_{t}\right)\right|^{2} d \mathcal{M}+2 \alpha \int_{0}^{T} E(t) d t
$$

where $\alpha$ is a positive constant. 
Estimate for $K_{3}:=\int_{0}^{T} \int_{\omega_{\varepsilon}} u\left(\nabla_{T} u \cdot \nabla_{T} \eta_{\varepsilon}\right) d \mathcal{M} d t$.

Considering (4.24) and applying the Cauchy-Schwarz inequality, we can write

$$
\begin{aligned}
\left|K_{3}\right| & \leq \frac{1}{2} \int_{0}^{T}\left[\int_{\omega_{\varepsilon}} \eta_{\varepsilon}\left|\nabla_{T} u\right|^{2} d \mathcal{M}+\int_{\omega_{\varepsilon}} \frac{\left|\nabla_{T} \eta_{\varepsilon}\right|^{2}}{\eta_{\varepsilon}}|u|^{2} d \mathcal{M}\right] d t \\
& \leq \frac{1}{2} \int_{0}^{T}\left[\int_{\omega_{\varepsilon}} \eta_{\varepsilon}\left|\nabla_{T} u\right|^{2} d \mathcal{M}+\frac{M}{\varepsilon^{2}} \int_{\omega_{\varepsilon}}|u|^{2} d \mathcal{M}\right] d t .
\end{aligned}
$$

Combining (4.25)-(4.28) we arrive at the following inequality:

$$
\begin{aligned}
\frac{1}{2} \int_{0}^{T} \int_{\omega_{\varepsilon}} \eta_{\varepsilon}\left|\nabla_{T} u\right|^{2} d \mathcal{M} d t \leq & |\mathcal{Y}|+\frac{\lambda_{1}^{-1}\|a\|_{L^{\infty}(\mathcal{M})}}{4 \alpha} \int_{0}^{T} \int_{\mathcal{M}} a(x)\left|g\left(u_{t}\right)\right|^{2} d \mathcal{M} \\
& +2 \alpha \int_{0}^{T} E(t) d t+\frac{M}{2 \varepsilon^{2}} \int_{0}^{T} \int_{\omega_{\varepsilon}}|u|^{2} d \mathcal{M} d t \\
& +a_{0}^{-1} \int_{0}^{T} \int_{\mathcal{M}} a(x) u_{t}^{2} d \mathcal{M} d t
\end{aligned}
$$

where

$$
\mathcal{Y}:=-\left[\int_{\omega_{\varepsilon}} u_{t} u \eta_{\varepsilon} d \mathcal{M}\right]_{0}^{T}
$$

Thus, combining (4.29) and (4.23), having in mind that

$$
\frac{1}{2} \int_{0}^{T} \int_{\mathcal{M} \backslash V}\left|\nabla_{T} u\right|^{2} d \mathcal{M} d t \leq \frac{1}{2} \int_{0}^{T} \int_{\omega_{\varepsilon}} \eta_{\varepsilon}\left|\nabla_{T} u\right|^{2} d \mathcal{M} d t
$$

and choosing $\alpha$ small enough, we deduce

$$
\begin{aligned}
\int_{0}^{T} E(t) d t \leq & |\chi|+C_{1}|\mathcal{Y}| \\
& +C_{2} \int_{0}^{T} \int_{\mathcal{M}}\left[a(x)\left|g\left(u_{t}\right)\right|^{2}+a(x)\left|u_{t}\right|^{2}\right] d \mathcal{M} d t \\
& +\frac{M C_{2}}{\varepsilon^{2}} \int_{0}^{T} \int_{\omega_{\varepsilon}}|u|^{2} d \mathcal{M} d t
\end{aligned}
$$

where $C_{2}=C_{2}\left(C_{1}, \lambda_{1}^{-1},\|a\|_{L^{\infty}(\mathcal{M})}, a_{0}^{-1}\right)$.

On the other hand, from (4.19), (4.30) and (2.8) the following estimate holds:

$$
\begin{aligned}
|\chi|+2 C_{2}|\mathcal{Y}| & \leq C(E(0)+E(T)) \\
& =C\left[2 E(T)+\int_{0}^{T} \int_{\mathcal{M}} a(x) g\left(u_{t}\right) u_{t} d \mathcal{M}\right]
\end{aligned}
$$

where $C$ is a positive constant which depends on $R$. 
Then, (4.31) and (4.32) yield

$$
\begin{aligned}
T E(T) & \leq \int_{0}^{T} E(t) d t \\
& \leq C E(T)+C\left[\int_{0}^{T} \int_{\mathcal{M}}\left[a(x)\left|g\left(u_{t}\right)\right|^{2}+a(x)\left|u_{t}\right|^{2}\right] d \mathcal{M} d t\right] \\
& +C \int_{0}^{T} \int_{\omega_{\varepsilon}}|u|^{2} d \mathcal{M} d t
\end{aligned}
$$

where $C$ is a positive constant which depends on $a_{0}, \lambda_{1}, R,\|a\|_{L^{\infty}(\mathcal{M})}, n$ and $\frac{M}{\varepsilon^{2}}$.

Our aim is to absorb the last term on the RHS of (4.33). In order to do this, let us consider the following lemma, where $T_{0}$ is a positive constant which is sufficiently large for our purposes.

Lemma 4.3. Under the hypotheses of Theorem 3.1 and for all $T>T_{0}$, there exists a positive constant $C\left(T_{0}, E(0)\right)$ such that if $\left(u, u_{t}\right)$ is the solution of (1.1) with weak initial data, we have

$$
\left.\int_{0}^{T} \int_{\mathcal{M}}|u|^{2} d \mathcal{M} d t \leq C\left(T_{0}, E(0)\right)\left\{\int_{0}^{T} \int_{\mathcal{M}}\left(a(x) g^{2}\left(u_{t}\right)\right)+a(x) u_{t}^{2}\right) d \mathcal{M} d t\right\} .
$$

Proof. We argue by contradiction exactly as in Lasiecka and Tataru's work [LA-TA]. For simplicity we shall denote $u^{\prime}:=u_{t}$. Let us suppose that (4.34) is not satisfied and let $\left\{u_{k}(0), u_{k}^{\prime}(0)\right\}$ be a sequence of initial data where the corresponding solutions $\left\{u_{k}\right\}_{k \in \mathbb{N}}$ of (1.1), with $E_{k}(0)$ assumed uniformly bounded in $k$, satisfy

$$
\lim _{k \rightarrow+\infty} \frac{\int_{0}^{T}\left\|u_{k}(t)\right\|_{L^{2}(\mathcal{M})}^{2} d t}{\int_{0}^{T} \int_{\mathcal{M}}\left(a(x) g^{2}\left(u_{k}^{\prime}\right)+a(x) u_{k}^{\prime 2}\right) d \mathcal{M} d t}=+\infty
$$

that is,

$$
\lim _{k \rightarrow+\infty} \frac{\int_{0}^{T} \int_{\mathcal{M}}\left(a(x) g^{2}\left(u_{k}^{\prime}\right)+a(x) u_{k}^{\prime 2}\right) d \mathcal{M} d t}{\int_{0}^{T}\left\|u_{k}(t)\right\|_{L^{2}(\mathcal{M})}^{2} d t}=0 .
$$

Since $E_{k}(t) \leq E_{k}(0) \leq L$, where $L$ is a positive constant, we obtain a subsequence, still denoted by $\left\{u_{k}\right\}$ from now on, which satisfies the convergence

$$
\begin{aligned}
& u_{k} \rightarrow u \text { weakly in } H^{1}\left(\Sigma_{T}\right), \\
& u_{k} \rightarrow u \text { weak star in } L^{\infty}(0, T ; V), \\
& u_{k}^{\prime} \rightarrow u^{\prime} \text { weak star in } L^{\infty}\left(0, T ; L^{2}(\mathcal{M})\right) .
\end{aligned}
$$

Employing compactness results we also deduce that

$$
u_{k} \rightarrow u \text { strongly in } L^{2}\left(0, T ; L^{2}(\mathcal{M})\right) .
$$

At this point we will divide our proof into two cases, namely, $u \neq 0$ and $u=0$.

(i) Case (I): $u \neq 0$.

We also observe that from (4.36) and (4.40) we have

$$
\lim _{k \rightarrow+\infty} \int_{0}^{T} \int_{\mathcal{M}}\left(a(x) g^{2}\left(u_{k}^{\prime}\right)+a(x) u_{k}^{\prime 2}\right) d \mathcal{M} d t=0 .
$$


Passing to the limit in the equation, when $k \rightarrow+\infty$, we get

$$
\left\{\begin{aligned}
u_{t t}-\Delta_{\mathcal{M}} u & =0 \text { on } \mathcal{M} \times(0, T), \\
u_{t} & =0 \text { on } \mathcal{M}_{*} \times(0, T),
\end{aligned}\right.
$$

and for $u_{t}=v$, we obtain, in the distributional sense,

$$
\left\{\begin{aligned}
v_{t t}-\Delta_{\mathcal{M}} v & =0 \text { on } \mathcal{M} \times(0, T) \\
v & =0 \text { on } \mathcal{M}_{*} \times(0, T) .
\end{aligned}\right.
$$

From uniqueness results due to Triggiani and Yao [TRI-YAO we conclude that $v \equiv 0$, that is, $u_{t}=0$. Indeed, let $V_{i}$ be as in Theorem 4.1 and $\Gamma=\partial V_{i}$, which is a smooth curve contained in $\mathcal{M}_{*}$. Since $v \equiv 0$ on $\mathcal{M}_{*}$ we deduce that $v=\partial_{\nu} v=0$ on $\Gamma$. Employing Triggiani and Yao's uniqueness results to the compact manifold $\bar{V}_{i}$ with boundary $\Gamma$ we infer that $v \equiv 0$ on $\bar{V}_{i}$, for each $i=1, \ldots, k$. Therefore, $v \equiv 0$ on $\mathcal{M}$ as we desired to prove. Returning to (4.42) we obtain the following elliptic equation a.e. in $(0, T)$ given by

$$
\left\{\begin{aligned}
\Delta_{\mathcal{M}} u & =0 \text { on } \mathcal{M} \\
u_{t} & =0 \text { on } \mathcal{M}
\end{aligned}\right.
$$

which implies that $u=0$, which is a contradiction.

(ii) Case (II): $u=0$.

Defining

$$
c_{k}:=\left[\int_{0}^{T} \int_{\mathcal{M}}\left|u_{k}\right|^{2} d \mathcal{M} d t\right]^{1 / 2}
$$

and

$$
\bar{u}_{k}:=\frac{1}{c_{k}} u_{k},
$$

we obtain

$$
\int_{0}^{T} \int_{\mathcal{M}}\left|\bar{u}_{k}\right|^{2} d \mathcal{M} d t=\int_{0}^{T} \int_{\mathcal{M}} \frac{\left|u_{k}\right|^{2}}{c_{k}^{2}} d \mathcal{M} d t=\frac{1}{c_{k}^{2}} \int_{0}^{T} \int_{\mathcal{M}}\left|u_{k}\right|^{2} d \mathcal{M} d t=1
$$

Setting

$$
\bar{E}_{k}(t):=\frac{1}{2} \int_{\mathcal{M}}\left|\bar{u}_{k}^{\prime}\right|^{2} d \mathcal{M}+\frac{1}{2} \int_{\mathcal{M}}\left|\nabla \bar{u}_{k}\right|^{2} d \mathcal{M},
$$

we deduce that

$$
\bar{E}_{k}(t)=\frac{E_{k}(t)}{c_{k}^{2}} .
$$

Recalling (4.33) we obtain, for $T$ large enough, that

$$
E(T) \leq \hat{C}\left[\int_{0}^{T} \int_{\mathcal{M}}\left(a(x) g^{2}\left(u_{t}\right)+a(x) u_{t}^{2}\right) d \mathcal{M} d t+\int_{0}^{T} \int_{\mathcal{M}}|u|^{2} d \mathcal{M} d t\right],
$$

and employing the identity $E(T)-E(0)=-\int_{0}^{T} \int_{\mathcal{M}} a(x) g\left(u_{t}\right) u_{t} d \mathcal{M} d t$, we get $E(t) \leq E(0) \leq \tilde{C}\left[\int_{0}^{T} \int_{\mathcal{M}}\left(a(x) g^{2}\left(u_{t}\right)+a(x) u_{t}^{2}\right) d \mathcal{M} d t+\int_{0}^{T} \int_{\mathcal{M}}|u|^{2} d \mathcal{M} d t\right]$, 
for all $t \in(0, T)$, where $T$ is sufficiently large. The last inequality and (4.46) yield

$$
\bar{E}_{k}(t):=\frac{E_{k}(t)}{c_{k}^{2}} \leq \tilde{C}\left[\frac{\int_{0}^{T} \int_{\mathcal{M}}\left(a(x) g^{2}\left(u_{k}^{\prime}\right)+a(x) u_{k}^{\prime 2}\right)}{\int_{0}^{T} \int_{\mathcal{M}}\left|u_{k}\right|^{2} d \mathcal{M} d t}+1\right] .
$$

From (4.36) and (4.47) we conclude that there exists a positive constant $\hat{M}$ such that

$$
\bar{E}_{k}(t):=\frac{E_{k}(t)}{c_{k}^{2}} \leq \hat{M}, \text { for all } t \in[0, T] \text { and for all } k \in \mathbb{N},
$$

that is,

$\frac{1}{2} \int_{\mathcal{M}}\left|\bar{u}_{k}^{\prime}\right|^{2} d \mathcal{M}+\frac{1}{2} \int_{\Omega}\left|\nabla \bar{u}_{k}\right|^{2} d \mathcal{M} \leq \hat{M}$, for all $t \in[0, T]$ and for all $k \in \mathbb{N}$.

For a subsequence $\left\{\bar{u}_{k}\right\}$, we obtain

$$
\begin{aligned}
& \bar{u}_{k} \rightarrow \bar{u} \text { weak star in } L^{\infty}(0, T ; V), \\
& \bar{u}_{k}^{\prime} \rightarrow \bar{u}^{\prime} \text { weak star in } L^{\infty}\left(0, T ; L^{2}(\mathcal{M})\right), \\
& \bar{u}_{k} \rightarrow \bar{u} \text { strongly in } L^{2}\left(0, T ; L^{2}(\mathcal{M})\right) .
\end{aligned}
$$

We observe that from (4.36) we deduce

$$
\lim _{k \rightarrow+\infty} \int_{0}^{T} \int_{\mathcal{M}} \frac{a(x) g^{2}\left(u_{k}^{\prime}\right)}{c_{k}^{2}} d \mathcal{M} d t=0 \text { and } \lim _{k \rightarrow+\infty} \int_{0}^{T} \int_{\mathcal{M}} a(x)\left|\bar{u}_{k}^{\prime}\right|^{2} d \mathcal{M} d t=0 .
$$

In addition, $\bar{u}_{k}$ satisfies the equation

$$
\bar{u}_{k}^{\prime \prime}-\Delta_{\mathcal{M}} \bar{u}_{k}+a(x) \frac{g\left(u_{k}^{\prime}\right)}{c_{k}}=0 \quad \text { on } \mathcal{M} \times(0, T) .
$$

Passing to the limit when $k \rightarrow+\infty$ and taking the above convergences into account, we obtain

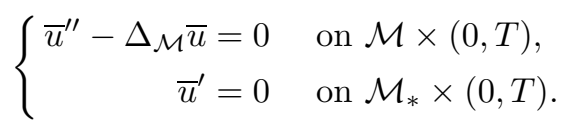

Then, $v=\bar{u}_{t}$ satisfies, in the distributional sense,

$$
\left\{\begin{aligned}
v_{t t}-\Delta_{\mathcal{M}} v & =0 \text { on } \mathcal{M} \\
v & =0 \text { on } \mathcal{M}_{*} .
\end{aligned}\right.
$$

Applying, again, uniqueness results due to Triggiani and Yao TRI-YAO, it follows that $v=\bar{u}_{t}=0$. Returning to (4.53) we have a.e. in $(0, T)$ that

$$
\left\{\begin{aligned}
\Delta_{\mathcal{M}} \bar{u} & =0 \text { on } \mathcal{M} \\
\bar{u}_{t} & =0 \text { on } \mathcal{M}
\end{aligned}\right.
$$

We deduce that $\bar{u}=0$, which is a contradiction in view of (4.45) and (4.51). The lemma is settled.

Inequalities (4.33) and (4.34) lead us to the following result.

Proposition 5.2.2. For $T>0$ large enough, the solution $\left[u, u_{t}\right]$ of (2.1) satisfies

$$
E(T) \leq C \int_{0}^{T} \int_{\mathcal{M}}\left[a(x)\left|u_{t}\right|^{2}+a(x)\left|g\left(u_{t}\right)\right|^{2}\right] d \mathcal{M} d t,
$$

where the constant $C=C\left(T_{0}, E(0), C, a_{0}, \lambda_{1}, R,\|a\|_{L^{\infty}(\mathcal{M})}, n, \frac{M}{\varepsilon^{2}}\right)$. 
4.5. Conclusion of Theorem 3.1. In what follows we will proceed exactly as in Lasiecka and Tataru's work [LA-TA] (see Lemma 3.2 and Lemma 3.3 of the referred paper) adapted to our context. Let $\Sigma:=\mathcal{M} \times(0, T)$,

$$
\begin{aligned}
\Sigma_{\alpha} & =\left\{(t, x) \in \Sigma:\left|u_{t}\right|>1 \text { a.e. }\right\} \\
\Sigma_{\beta} & =\Sigma \backslash \Sigma_{\alpha} .
\end{aligned}
$$

Then using hypothesis (iii) in Assumption 2.1, we obtain

$$
\int_{\Sigma_{\alpha}} a(x)\left(\left[g\left(u_{t}\right)\right]^{2}+\left(u_{t}\right)^{2}\right) d \Sigma_{\alpha} \leq\left(k^{-1}+K\right) \int_{\Sigma_{\alpha}} a(x) g\left(u_{t}\right) u_{t} d \Sigma_{\alpha}
$$

Moreover, from (3.1),

$$
\int_{\Sigma_{\beta}} a(x)\left(\left[g\left(u_{t}\right)\right]^{2}+\left(u_{t}\right)^{2}\right) d \Sigma_{\beta} \leq\left(1+\|a\|_{\infty}\right) \int_{\Sigma_{\beta}} h\left(a(x) g\left(u_{t}\right) u_{t}\right) d \Sigma_{\beta} .
$$

Then, by Jensen's inequality,

$$
\begin{aligned}
(1+ & \left.\|a\|_{\infty}\right) \int_{\Sigma_{\beta}} h\left(g\left(u_{t}\right) u_{t}\right) d \Sigma_{\beta} \\
& \leq\left(1+\|a\|_{\infty}\right) \text { meas }(\Sigma) h\left(\frac{1}{\text { meas }(\Sigma)} \int_{\Sigma} a(x) g\left(u_{t}\right) u_{t} d \Sigma\right) \\
& =\left(1+\|a\|_{\infty}\right) \text { meas }(\Sigma) r\left(\int_{\Sigma} a(x) g\left(u_{t}\right) u_{t} d \Sigma\right)
\end{aligned}
$$

where $r(s)=h\left(\frac{s}{\text { meas }(\Sigma)}\right)$ is defined in (3.2). Thus

$$
\begin{array}{ll}
\int_{\Sigma} a(x)\left(\left[g\left(u_{t}\right)\right]^{2}+\left(u_{t}\right)^{2}\right) d \Sigma \leq & \left(k^{-1}+K\right) \int_{\Sigma} a(x) g\left(u_{t}\right) u_{t} d \Sigma \\
& +\left(1+\|a\|_{\infty}\right) \text { meas }(\Sigma) r\left(\int_{\Sigma} a(x) g\left(u_{t}\right) u_{t} d \Sigma\right) .
\end{array}
$$

Splicing together (4.54) and (4.58), we have

$$
\begin{aligned}
E(T) \leq & \left(1+\|a\|_{\infty}\right) C\left[\frac{K_{0}}{\left(1+\|a\|_{\infty}\right)} \int_{\Sigma} a(x) g\left(u_{t}\right) u_{t} d \Sigma\right. \\
& \left.+ \text { meas }(\Sigma) r\left(\int_{\Sigma} a(x) g\left(u_{t}\right) u_{t} d \Sigma\right)\right]
\end{aligned}
$$

where $K_{0}=k^{-1}+K$. Setting

$$
\begin{aligned}
L & =\frac{1}{\text { Cmeas }(\Sigma)\left(1+\|a\|_{\infty}\right)}, \\
c & =\frac{K_{0}}{\text { meas }(\Sigma)\left(1+\|a\|_{\infty}\right)},
\end{aligned}
$$

we obtain

$$
p[E(T)] \leq \int_{\Sigma} a(x) g\left(u_{t}\right) u_{t} d \Sigma=E(0)-E(T)
$$

where the function $p$ is as defined in (3.3). To finish the proof of Theorem 3.1, we invoke the following result from I. Lasiecka et al. [LA-TA]. 
Lemma B. Let $p$ be a positive, increasing function such that $p(0)=0$. Since $p$ is increasing we can define an increasing function $q, q(x)=x-(I+p)^{-1}(x)$. Consider a sequence $s_{n}$ of positive numbers which satisfies

$$
s_{m+1}+p\left(s_{m+1}\right) \leq s_{m} .
$$

Then $s_{m} \leq S(m)$, where $S(t)$ is a solution of the differential equation

$$
\frac{d}{d t} S(t)+q(S(t))=0, \quad S(0)=s_{0} .
$$

Moreover, if $p(x)>0$ for $x>0$, then $\lim _{t \rightarrow \infty} S(t)=0$.

Taking into account the above result, we replace $T$ (resp. 0) in (4.60) with $m(T+1)$ (resp. $m T)$ in order to get

$$
E(m(T+1))+p(E(m(T+1))) \leq E(m T), \text { for } m=0,1, \ldots
$$

Applying Lemma B with $s_{m}=E(m T)$ results in

$$
E(m T) \leq S(m), \quad m=0,1, \ldots
$$

Finally, using the inherent dissipativity of $E(t)$ given in relation (2.8), we have for $t=m T+\tau, 0 \leq \tau \leq T$,

$$
E(t) \leq E(m T) \leq S(m) \leq S\left(\frac{t-\tau}{T}\right) \leq S\left(\frac{t}{T}-1\right) \text { for } t>T,
$$

where we have used the fact that $S($.$) is dissipative. The proof of Theorem 3.1$ is now completed.

\section{ACKNOWLEDGEMENTS}

The authors would like to thank Professor Roberto Triggiani for his kind attention and helpful remarks during the period when this paper was written.

\section{REFERENCES}

[ALA $] \quad$ F. Alabau-Boussouira, Convexity and weighted integral inequalities for energy decay rates of nonlinear dissipative hyperbolic systems, Appl. Math. Optim. 51(1), (2005), 61-105. MR2101382 (2005h:34149)

[An1] D. Andrade, M. M. Cavalcanti, V. N. Domingos Cavalcanti, and H. P. Oquendo. Existence and asymptotic stability for viscoelastic evolution problems on compact manifolds. J. Comput. Anal. Appl., vol. 8, no. 3, pp. 173-193, 2006. MR2225653 (2006k:35032)

[An2] D. Andrade, M. M. Cavalcanti, V. N. Domingos Cavalcanti, and H. P. Oquendo. Existence and asymptotic stability for viscoelastic evolution problems on compact manifolds (part II). J. Comput. Anal. Appl, vol. 8, no. 3, pp. 287-301, 2006. MR:2223868(2006m:35036)

[BAR] C. Bardos, G. Lebeau and J. Rauch, Contrôle et stabilisation de l'équation des ondes, Appendix II in J. L. Lions Contrôlabilité exacte des systèmes distribués, Collection RMA, Vol. 8, Masson, Paris, 1988. MR 953547 (90a:49040)

[CA-DO] M. M. Cavalcanti and V. N. Domingos Cavalcanti, Existence and asymptotic stability for evolution problems on manifolds with damping and source terms, $J$. Math. Anal. Appl. 291(1), (2004), 109-127. MR2034061 (2005h:35238)

[Ca-Do-Fu-So] M. M. Cavalcanti, V. N. Domingos Cavalcanti, R. Fukuoka and J. A. Soriano, Uniform stabilization of the wave equation on compact surfaces and locally distributed damping. To appear in Methods Appl. Anal.

[CAVAL] M. M. Cavalcanti and H. P. Oquendo, Frictional versus viscoelastic damping in a semilinear wave equation. SIAM J. Control Optim. 42 (2003), no. 4, 1310-1324. MR2044797 (2005d:35257) 
[CA-DO-LA] M. M. Cavalcanti, V. N. Domingos Cavalcanti and I. Lasiecka, Wellposedness and optimal decay rates for wave equation with nonlinear boundary damping-source interaction, Journal of Differential Equations, 236 (2007), 407-459. MR2322019 (2008c:35189)

[CHR] H. Christianson, Semiclassical non-concentration near hyperbolic orbits, J. Funct. Anal. 246 (2007), no. 2, 145-195. MR2321040

[Do Carmo] M. P. do Carmo, Riemannian geometry. Mathematics: Theory \& Applications. Birkhäuser Boston, Inc., Boston, MA, 1992. MR.1138207 (92i:53001)

[HIT] M. Hitrik, Expansions and eigenfrequencies for damped wave equations, Journées "Équations aux Dérivées Partielles" (Plestin-les-Grèves, 2001), Exp. No. VI, 10 pp., Univ. Nantes, Nantes, 2001. MR 1843407 (2002h:58050)

[LA-TA $] \quad$ I. Lasiecka and D. Tataru, Uniform boundary stabilization of semilinear wave equation with nonlinear boundary damping, Differential and Integral Equations, 6 (1993), 507-533. MR.1202555 (94c:35129)

[LA-TRI] I. Lasiecka and R. Triggiani, Uniform stabilization of the wave equation with Derichlet or Neumann feedback control without geometric conditions, Appl. Math. Optim., 25 (1992), 189-124 MR.1142681 (93b:93099)

[LIONS1] J. L. Lions, Contrôlabilité exacte, perturbations et stabilisation de systèmes distribués, tome 1, Masson, 1988. MR0953547 (90a:49040)

[LIONS2] J. L. Lions, Contrôlabilité exacte, perturbations et stabilisation de systèmes distribués, tome 2, Masson, 1988. MR0963060 (89k:93017)

[LiMa] J.L.Lions-E.Magenes, Problèmes Aux Limites Non Homogènes et Applications, Dunod, Paris, 1968, Vol. 1.

[Ra-Ta] J. Rauch and M. Taylor, Decay of solutions to nondissipative hyperbolic systems on compact manifolds. Comm. Pure Appl. Math. 28(4) (1975), 501-523 . MR0397184 $(53: 1044 \mathrm{a})$

[Ta1] D. Tataru, Unique continuation for solutions to PDE's: Between Holmgren's theorem and Hörmander's theorem. Comm. Partial Differential Equations 20 (1995), no. 5-6, 855-884. MR1326909 (96e:35019)

[Ta2] D. Tataru. Unique continuation for operators with partially analytic coefficients. J. Math. Pures Appl. (9) 78 (1999), no. 5, 505-521. MR1697040(2000e:35005)

[Tou] D. Toundykov, Optimal decay rates for solutions of a nonlinear wave equation with localized nonlinear dissipation of unrestricted growth and critical exponent source terms under mixed boundary conditions, Nonlinear Analysis T. M. A. 67 (2007), 514-544. MR2317185 (2008f:35257)

[TRI-YAO] R. Triggiani and P. F. Yao, Carleman estimates with no lower-order terms for general Riemannian wave equations. Global uniqueness and observability in one shot, Appl. Math. and Optim. 46 (Sept./Dec. 2002), 331-375. Special issue dedicated to J. L. Lions. MR1944764 (2003j:93042)

Department of Mathematics, State University of Maringá, 87020-900, Maringá, PR, BRAZIL

Department of Mathematics, State University of Maringá, 87020-900, Maringá, PR, BRAZIL

Department of Mathematics, State University of Maringá, 87020-900, Maringá, PR, BRAZIL

Department of Mathematics, State University of Maringá, 87020-900, Maringá, PR, BRAZIL 